\title{
Annals of Case Reports
}

\section{Do we Need Laparatomy in Every Pnuemoperitoneum Xrs?}

\section{Haitham Akram Saimeh"}

King Faisal Speciality Hospital and Research Centre, Saudi Arabia

"Corresponding author: Haitham Akram Saimeh, King Faisal Speciality Hospital and Research Centre, Saudi Arabia

Citation: Saimeh HA. (2021) Do we Need Laparatomy in Every Pnuemoperitoneum Xrs? Ann Case Report 6: 694. DOI: $10.29011 / 2574-7754.100694$

Received Date: 11 August, 2021; Accepted Date: 16 August, 2021; Published Date: 20 August, 2021

\section{Introduction}

The presence of free air within the peritoneal cavity is referred as pneumoperitoneum, there are several causes which lead to it most commonly perforated abdominal organs mainly perforated peptic ulcers, other causes include penetrating traumatic injury, inflammatory conditions such as mega colon [1]. In over $90 \%$ of the patients the presence of intraperitoneal free air is a sign of hollow viscus perforation. On the other hand, the presence of pneumoperitoneum does not always indicate intraabdominal perforation therefore may not always be an indication fore laparotomy. The main aim of this case report is to raise awareness that each case of pneumoperitoneum should be individualized taking into consideration if any of the etiological factors are present, in order to avoid performing unnecessary laparotomy.

\section{Case}

A 64-year-old gentle man, known case of sacral chondroma that perforated many times together with consistent recurrences, with multiple time surgical excision and palliative radiotherapy treatment. The patient presented to the emergency department with picture of abdominal pain and vomiting, without constipation and fever. On physical examination, the patient was conscious and oriented, heart rate: 110 beats per minute, temperature: 37 Celsius, and blood pressure: 136/71. There was tenderness on the upper abdomen and general abdominal distention. In the rectum, there was soft stool and blood was seen on the gloves. Hard mass was felt at the buttock area at 3 o'clock. Abdominal CT-scan was done and showed diffuse dilatation of the bowel loops together with pneumoperitoneum, suggestive of hallow viscus perforation. Multidisciplinary team assessment and impression concluded that the patient was currently stable and having a perforated viscus. The management plan set was to admit the patient, place him on nasogastric tube, NPO, blood culture was done, and cross match of 2 units of packed red blood cells were ordered to prepare for operating theatre the next morning. The patient was taken to the operating room for laparotomy, the findings of the greater omentum pneumoperitoneum, no perforated viscus with possible rupture of one of the bullae giving the picture of pneumoperitoneum

\section{Discussion}

As previously mentioned, pneumoperitoneum is referred as the presence of air within the peritoneal cavity. There are two types of pneumoperitonea, spontaneous pneumoperitoneum which is characterized by intraperitoneal gas that's not associated with gastrointestinal tract perforation and no clear aetiology being identified. On the other hand, surgical pneumoperitoneum usually indication of bowel perforation which requires an emergent exploratory laparotomy. Surgical pneumoperitoneum constitutes $85-90 \%$ of all pneumoperitoneum cases, on the other hand nonsurgical pneumoperitoneum represents $5-15 \%$ of all cases $[2,3]$.

Medicine physicians together with surgeons should assess the patient clinical history and do appropriate and adequate physical abdominal examination to avoid carrying any unnecessary laparotomy. If signs and symptoms of peritonitis are absent conservative management is preferred to avoid the burden and consequences of unnecessary surgery. Regarding our case, in addition to the CT-scan report, the patient clinical presentation did require surgical intervention, therefore conservative treatment in such case presentation was not considered as a good management plan to be set, since there was reasonable surgical etiological cause that could be recognized.

Below are attached CT-scans that showed dilated bowel loops and dilated transverse colon respectively. 
Citation: Saimeh HA. (2021) Do we Need Laparatomy in Every Pnuemoperitoneum Xrs? Ann Case Report 6: 694. DOI: 10.29011/25747754.100694

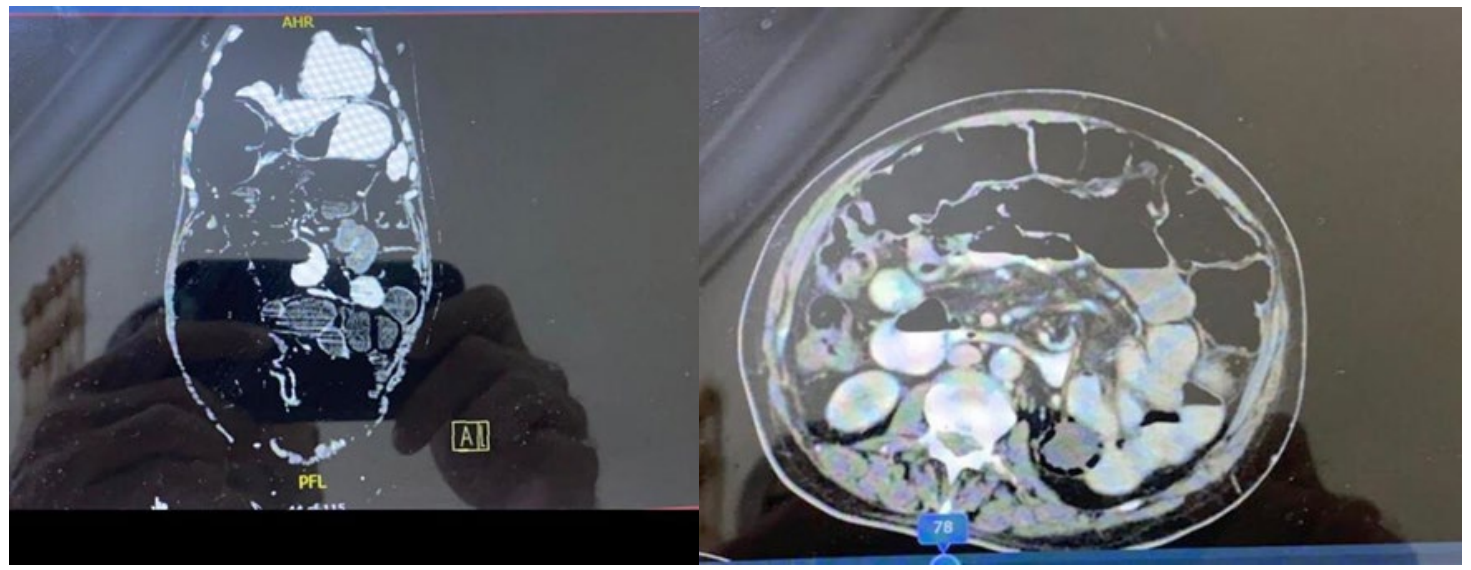

Figure 1: CT-scans that showed dilated bowel loops and dilated transverse colon respectively.

\section{Conclusion}

Appreciation of each surgical case together with the individualized case risk factors should raise awareness to reduce carrying unnecessary laparotomy. Therefore, detailed history in addition to clinical physical examination should be done before setting the management plan.

\section{References}

1. Makki AM. (2017) The Pattern of Causes of Pneumoperitoneuminduced Peritonitis: Results of an Empirical Study, Journal of Microscopy and Ultrastructure, 5: 28-31.
2. Williams NM, Watkin DF. (1997) Spontaneous pneumoperitoneum and other nonsurgical causes of intraperitoneal free gas. Postgrad Med J. 73: $531-537$.

3. Tuveri M, Borsezio V, Melis G, Muntoni G, Gabbas A, et al. (2006) Pneumoperitoneo: è sempre indicata la laparotomia esplorativa? [Pneumoperitoneum: is exploratory laparotomy always indicated?]. Chir Ital. 58: 611-618. 